\title{
mHealth to reduce HIV-related stigma among youth in the United States: a scoping review
}

\author{
Marta I. Mulawa ${ }^{1,2}$, A. Lina Rosengren ${ }^{3}$, K. Rivet Amico ${ }^{4}$, Lisa B. Hightow-Weidman ${ }^{3}$, Kathryn E. Muessig ${ }^{5}$ \\ ${ }^{1}$ School of Nursing, Duke University, Durham, NC, USA; ${ }^{2}$ Duke Global Health Institute, Duke University, Durham, NC, USA; ${ }^{3}$ Institute for Global \\ Health and Infectious Diseases, University of North Carolina at Chapel Hill, Chapel Hill, NC, USA; ${ }^{4}$ Department of Health Behavior and Health \\ Education, School of Public Health, University of Michigan, Ann Arbor, MI, USA; ${ }^{5}$ Department of Health Behavior, Gillings School of Global \\ Public Health, University of North Carolina at Chapel Hill, Chapel Hill, NC, USA \\ Contributions: (I) Conception and design: K Muessig, R Amico, L Hightow-Weidman; (II) Administrative support: None; (III) Provision of study \\ materials or patients: None; (IV) Collection and assembly of data: M Mulawa, L Hightow-Weidman; (V) Data analysis and interpretation: All \\ authors; (VI) Manuscript writing: All authors; (VII) Final approval of manuscript: All authors. \\ Correspondence to: Marta I. Mulawa, PhD, MHS. School of Nursing, Duke University, DUMC 3322, 307 Trent Drive, Durham, NC 27710, USA. \\ Email: marta.mulawa@duke.edu.
}

\begin{abstract}
Multiple intersecting stigmas and discrimination related to sex, gender, HIV, and race/ethnicity may challenge HIV prevention and treatment service utilization, particularly among youth. This scoping review describes recent and ongoing innovative mobile health (mHealth) interventions among youth in the United States that aim to reduce stigma as an outcome or as part of the intervention model. To identify examples of stigma-mitigation via mHealth, we searched peer-reviewed published literature using keyword strategies related to mHealth, HIV, stigma, and youth (ages 10 to 29). We identified eleven articles that met our inclusion criteria, including three describing data from two randomized controlled trials (RCTs), five describing pilot studies, one describing the process evaluation of an ongoing intervention, one describing formative work for intervention development, and one published study protocol for an ongoing intervention. We review these articles, grouped by HIV prevention and care continuum stages, and describe the mHealth approach used, including telehealth, simulation video games, motion comics, smartphone applications (apps), social media forums, online video campaigns, video vignettes, and a computerized behavioral learning module. Four studies focused on preventing primary acquisition through individual-level behavior change (e.g., reducing condomless anal intercourse), three focused on increasing HIV testing, three focused on linking to prevention services [e.g., pre-exposure prophylaxis (PrEP) and post-exposure prophylaxis (PEP)] and one focused on promoting adherence to antiretroviral therapy (ART). Our review did not identify any published studies using mHealth with a primary aim to reduce stigma as a way to improve care engagement and increase viral suppression among youth in the United States. Additional RCTs and implementation studies examining the effectiveness of mHealth stigma-reduction interventions on HIV-related outcomes are needed to end the HIV epidemic among youth. mHealth offers unique advantages to address the complex intersecting stigma barriers along the HIV continuum to improve HIV-related outcomes for youth.
\end{abstract}

Keywords: mHealth; youth; HIV; stigma; technology

Received: 19 February 2020; Accepted: 27 March 2020; Published: 20 April 2021.

doi: 10.21037/mhealth-20-68

View this article at: http://dx.doi.org/10.21037/mhealth-20-68

(C) mHealth. All rights reserved. 


\section{Introduction}

In the United States (US), youth ages 13 to 24 years account for $22 \%$ of new HIV diagnoses (1), are the least likely group to be linked to care (2), and only $25 \%$ of youth with HIV attain an undetectable viral load (3). The reasons for poor health outcomes for youth with HIV (YHIV) and elevated HIV incidence among youth are multifactorial, but stigma plays a significant role $(2,4-6)$. Stigma occurs at all points along the continuum of HIV prevention and care, acting as a barrier to HIV testing (4), uptake of pre-exposure prophylaxis $(\mathrm{PrEP})(7,8)$, linkage and retention in care $(9)$, and adherence to antiretroviral therapy (ART) (10-12). Stigma also impacts social determinants of prevention and treatment, including HIV status disclosure (13), sexual risk behavior (14,15), and mental health among YHIV (11,16-18).

Young men who have sex with men (MSM) and transgender women are at disproportionate risk for HIV and poor HIV-related health outcomes $(19,20)$, particularly within racial and ethnic minority groups. Of the 8,164 new cases of HIV in the US among youth in 2017, $81 \%$ occurred in MSM (20). Further, MSM of color made up over onethird of the new HIV diagnoses in 2017, three-quarters of those were in young Black MSM aged 13-24 (19). Stigma affecting young MSM of color is intersectional, amplifying disparities from multiple sources of stigma, including racial, sexual, and gender minority status (21) in addition to HIV-related stigma $(13,22,23)$. These intersecting stigmas contribute to poor outcomes at all points along the HIV prevention and care continuum among MSM of color compared to other groups in the US $(2,13,19)$. Gender minority youth also have a disproportionate incidence of HIV. Transgender women are three times more likely to receive an HIV diagnosis than the national average; with an HIV prevalence of $44 \%$ among transgender women of color (24). Transgender women's vulnerability to HIV is compounded by consequences of stigma related to their gender identity and expression, including violence, discrimination, and transphobia (25).

Stigma intervention development has been challenging due to the multidimensional and multi-level nature of stigma (26) and ineffectual means to comprehensively intervene with participants in sustainable ways that go beyond simple education or information giving. Implementation of HIV-stigma interventions that require in-person attendance can be hampered by intervention access including distance-barriers, particularly in resourcepoor settings (27). Participants currently experiencing or anticipating high levels of stigma may also face additional obstacles, including reduced resources, social isolation, and maladaptive coping responses including avoidance and substance use that may serve as additional barriers to participation (10). Two recent reviews of global HIVrelated stigma reduction interventions concluded that very few studies have focused on young people and other groups with an elevated risk of HIV, including MSM and transgender individuals $(28,29)$. The few interventions that have shown positive impact on reducing HIV/AIDS stigma among youth have primarily used education, including radio diaries, an "edutainment" soap opera series, and school-based approaches (dialogues, print materials, short movies) (29). The lack of widely accepted and validated stigma measures has also limited our ability to compare and contrast evaluated interventions $(30,31)$.

The rise of mobile health (mHealth) has presented new possibilities for intervention content and delivery, providing large populations with increased access to evidence-based interventions at potentially lower cost (32). For youth, mHealth stigma interventions could provide further advantages in delivery of multi-media interventions shown to be effective in reducing stigma among youth $(29,33)$, and there is high acceptability of mobile technologies among youth for delivery of health interventions (34). mHealth additionally offers advantages in addressing the complex structural and psychosocial barriers to HIV prevention and care, providing a platform for tailored stigma interventions that are culturally-appropriate and sensitive to individual needs $(35,36)$. Indeed, it is possible for mHealth interventions to dynamically tailor intervention content based on evolving user needs and preferences (37). This ability to offer tailored intervention content is particularly important in the context of intersectional stigma. mHealth approaches can also overcome barriers to service utilization by reaching participants directly (6), increase access to affirming health information, foster social support and resilience (38), and provide structured, developmentallyappropriate tools for building healthy habits (e.g., providing daily reminders or goal-setting activities to support PrEP or ART adherence). Finally, it is relevant to note that many youth in the US experience social connectedness through mobile technologies (39), thus facilitating contact with peers who may be experiencing similar stigmas could provide new and meaningful social support.

$\mathrm{mHealth}$ has been leveraged for interventions to reduce $\mathrm{HIV}$-related stigma, yet literature in this area is still scant (27) and the extent to which existing interventions have specifically 
engaged youth in stigma reduction efforts is unknown. This paper provides the first review of mHealth interventions for youth in the US that aimed, as an outcome or as part of the framing or conceptual model for the intervention, to reduce stigma as a way to improve HIV-related outcomes. While stigma is a barrier to HIV prevention and care across most countries (12), we limited our review to US-based studies in order to make more focused recommendations for mHealth approaches. Specifically, the contextual drivers and manifestations of stigma vary across settings (40) and likely require appropriately tailored mHealth intervention approaches. Further, although uptake of mobile technology is nearly universal, the use of specific platforms and tools differs across countries (e.g., broad use of WeChat in China or WhatsApp in South Africa). In this way, we can better inform the US adolescent and young adult HIV prevention and care agenda.

\section{Methods}

In February, 2020, we searched PubMed for English language publications using combinations of keywords and $\mathrm{MeSH}$ terms related to mHealth, HIV, stigma, and youth (see Supplementary File for full search strategy). Two coauthors (MM and $\mathrm{LH})$ independently assessed each title and abstract to identify relevant records for inclusion. Studies were excluded if they were clearly not meeting the inclusion criteria or if they were conducted outside the US. Full texts were pulled for all abstracts noted as relevant by at least one reviewer. Final decisions on article inclusion were made after reviewing the full-text of each potentially eligible article.

Studies were included if they (I) reported on the development, pilot testing, process evaluation, or randomized controlled trial (RCT) of an HIV-related intervention, (II) used mobile technology as a component of the intervention, (III) included stigma (broadly defined as including social stigma, shame, discrimination, prejudice, homophobia, transphobia, racism, etc.) as an outcome or as part of the framing or conceptual model for the intervention, and (IV) included adolescent or young adult populations (age 10-29 years). Given the wide-ranging and multi-faceted nature of stigma $(21,41)$, our review was intentionally broad in its operationalization of stigma and was not limited to a single form (e.g., internalized stigma) or level (e.g., structural stigma).

The following data were extracted from each article: study population (including inclusion criteria and age range and mean); sample size; setting; study design and methods; main focus of study; description of the intervention; theory or framework used; mHealth approach; types of stigma addressed; and key findings. A narrative summary was also prepared for each article describing key discussion points and the limitations noted by the authors.

\section{Results}

Among the 310 articles extracted, eleven met the inclusion criteria. The flow diagram for the scoping review is presented in Figure 1. The characteristics of the included studies are summarized in http://cdn.amegroups.cn/static/ application/6268d814493893bc2d077ecd1422572c/10.2 1037mhealth-20-68-1.pdf. The 11 included manuscripts included three articles describing data from two RCTs, four describing pilot studies, one describing the process evaluation of an ongoing intervention, one describing formative work for intervention development, and one published study protocol for an ongoing intervention. We categorized each manuscript into the following HIV prevention and care continuum categories: (I) preventing primary acquisition through individual-level behavior change (e.g., reducing condomless anal intercourse), (II) increasing HIV testing, (III) linking to prevention services (e.g., PrEP) and (IV) promoting adherence to ART. Within this continuum of care framework, we begin by highlighting evidence from RCTs followed by data from pilot/feasibility studies, formative research, and study protocols.

\section{Preventing primary acquisition through individual-level behavior change}

Four included articles $(38,42-44)$ were focused on preventing primary acquisition of HIV through individuallevel behavior change, mostly by promoting safer sexual behaviors and reducing condomless anal intercourse.

Three of the articles presented data from two RCTs, both of which aimed to reduce condomless anal intercourse among MSM. The first RCT, conducted by Christensen et al., evaluated the effectiveness of SOLVE (Socially Optimized Learning in Virtual Environments), a brief 30-minute simulation video game addressing sexual shame to reduce condomless anal intercourse with non-primary partners among HIV-negative MSM aged 18-24 years (43). The SOLVE intervention used virtual intelligent agents in a $3 \mathrm{D}$ animated serious game designed to simulate risky situations, allowing the participant to make decisions that 


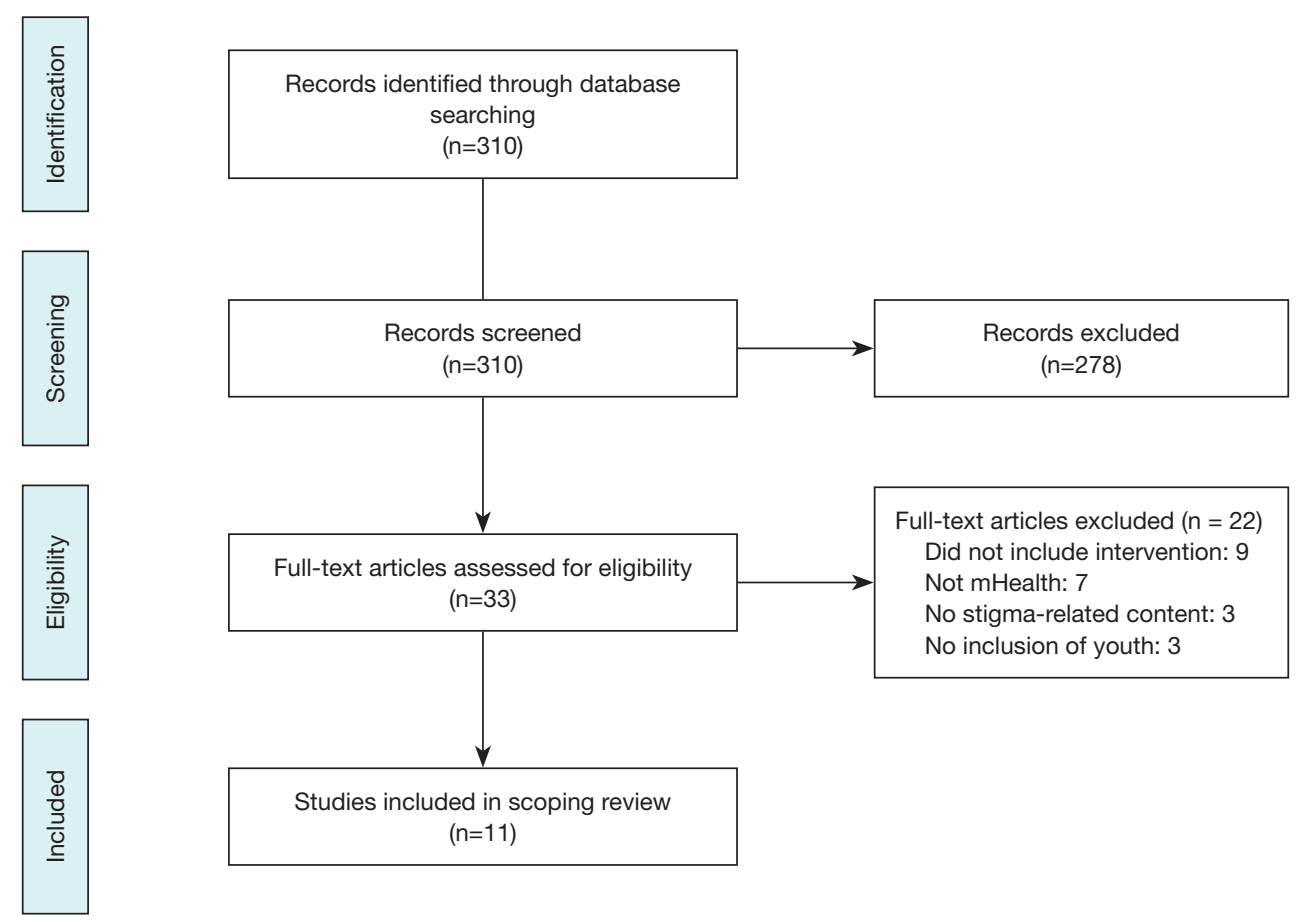

Figure 1 Flow diagram of articles included in scoping review.

shaped the narrative of the game and provided feedback. Participants randomized to the SOLVE treatment condition reported greater reductions in sexual shame immediately after the intervention, which in turn predicted reductions in condomless anal intercourse at the 3-month followup. The direct effect of the intervention on condomless anal intercourse was not significant (as it had been in previous SOLVE studies), and the authors state that they are conducting mediation analyses to examine potential suppressors of the intervention's effect (43).

The second RCT, which evaluated the effectiveness of HealthMpowerment (HMP), was the parent trial for the studies presented by Barry et al. and Bauermeister et al. $(38,42)$. HealthMpowerment (HMP) was a webbased intervention built using mobile responsive design that provided young MSM of color in the southeastern US, irrespective of HIV status, with a supportive online community. HMP was designed to build community and empower participants in order to reduce condomless anal intercourse (45). Bauermeister et al. coded all user-generated content created by participants within HMP (i.e., forum posts, anonymous questions to a doctor, and multimedia content shared with others) and used hierarchical linear modeling to examine the relationship between stigma- related user-generated content and longitudinal changes in stigma scores (42). The study found that user-generated posts regarding stigma were associated with participants' changes in sexuality- and HIV-related stigma over time. Barry et al. analyzed content posted in the forum as well as multimedia posts shared by HMP participants to understand how resilience processes occurred in response to stigma and discrimination (38). This study documented how peer-to-peer social support and empowering other youth through role modeling, and encouragement served to amplify resilience and buffer the impact of stigmatizing contexts on young MSM of color enrolled in the study.

Willis et al. conducted a one-arm pilot study evaluating a 38-minute, 3-episode motion comic series aimed at improving HIV/sexually transmitted infection (STI) knowledge attitudes, beliefs, and behavioral intentions related to sexual behavior among young people ages 15-24 (44). The storylines in the motion comics were created based on feedback from young people, and included topics related to HIV/STI stigma. Mixed-methods data (including HIV stigma scores and behavioral intentions to engage in safe sax) were collected through 15 focus group discussions in a pre-test, immediate posttest design. Participants in the pilot reported significantly lower HIV stigma and significantly 
higher behavioral intentions to engage in safe sex after viewing the motion comics. While the motion comics were not implemented using mobile technology for this pilot, the authors describe future plans to distribute the motion comics through multiple venues, including mobile phones, applications (apps), websites, and social media (44).

\section{Increasing HIV testing}

Three articles focused on increasing HIV testing, including one presenting the results of a pilot test (46) and two papers describing the protocol (47) and results (48) of a pilot RCT. The first article, by O'Donnell et al., reported on a community-based participatory research approach to the development and one-group pilot testing of Keep it Up (KIU), a 90-minute intervention designed to overcome the stigma associated with HIV and HIV testing by incorporated testing and prevention counseling into a broader health promotion campaign for young Black men (46). In addition to a social marketing campaign, KIU included a 45-minute computerized behavioral learning module which included videos and interactive skill-building games. The computerized module (delivered on laptop computers in private spaces) was designed with input from a community advisory board, expert advisors, and focus group participants, and was found to be acceptable, with $95 \%$ of participants positively endorsing the module as interesting and entertaining (46).

The protocol paper, by Stephenson et al., is for a pilot RCT of Project Moxie, a telehealth intervention designed to promote HIV testing among transgender and gender nonconforming youth ages 15-24 years (47). Project Moxie pairs HIV self-testing with remote video-based counseling (via a HIPPA-compliant video-chat session) and support from a trained, gender-affirming counselor. Given the many structural barriers youth may face to HIV testing, Project Moxie uses elements of motivational interviewing to explore barriers to HIV testing and provide solutions, explicitly including advice the counselor may give for participants reporting fears of stigma or discrimination as a barrier (47). Project Moxie was piloted with a diverse sample of 202 youth and the results revealed that youth who opted to participate in the video-chat counseling reported high levels of satisfaction with the intervention. Unfortunately, only $48 \%$ of the participants in the intervention arm opted to use this counseling session (48), with anecdotal data suggesting participants were worried about having to be seen physically by somebody they did not know. The authors discuss the importance of providing options to youth and acknowledging that youth may have differing needs and levels of comfort in engaging in various intervention components.

\section{Promoting biomedical prevention (e.g., PrEP and PEP)}

We identified three articles that were focused on promoting biomedical prevention services, including PrEP (49-51) and post-exposure prophylaxis (PEP) (52). The first was a onearm pilot, by Refugio et al. that explored the feasibility and acceptability of PrEPTECH, a telehealth-based initiation program for PrEP targeting young MSM of color (ages 1825 years) in the San Francisco Bay Area (49). The PrEPTECH intervention was developed using a youth-centered approach and the project website included customizable PrEP reminders that could be delivered through text or email. The authors noted that PrEPTECH addressed stigma by having counselors that were knowledgeable about PrEP and specific health issues affecting young MSM. At baseline, more than half of the participants reported feeling uncomfortable going to a doctor's office or clinic for PrEP. While more than half of the participants (52.4\%) reported hearing about others being stigmatized for taking PrEP at the 90-day follow-up (likely related to assumptions of increased risk-taking behavior while on PrEP), less than 15\% of PrEPTECH participants reported personally experiencing PrEP stigma (49).

The next study, by Thomann et al., described an evaluation of an ongoing, online video campaign designed to increase knowledge of PrEP as well as to address barriers related to PrEP adherence, including sex shaming (50). The study presents results from two focus groups with MSM and transgender women at risk for HIV (ages 21-50) in New York to assess their responses to this campaign. The campaign was advertised on social media and was viewed/ shared over 150,000 times between November, 2015 and January, 2016. Stigma emerged as an important theme in both of the focus groups, with participants describing how PrEP-related stigma could impact decision-making related to PrEP initiation (50).

Finally, there was an article by Schnall et al. on the development of MyPEEPS Mobile, a mobile responsedriven web-based platform targeting young MSM, ages 14-18 years (51). The MyPEEPS Mobile intervention includes intervention content on stigma, including how to handle "outness" and coming out. Additionally, it was 
noted that the panel recommended that the intervention include content on activating self-reflection about how stigma might influence personal decision-making. While the original MYPEEPS intervention focused on reducing sexual behavior while under the influence of substances, the advisory group providing guidance for the adaptation to younger MSM recommended including additional information on biomedical interventions (including PrEP, PEP, and home-based testing). Given the young age of participants, it was recommended that the MyPEEPS Mobile intervention provide basic information for these biomedical approaches along with information on how to access the services in the city (51).

\section{Promoting adberence to ART}

Only one article, by Mimiaga et al., focused on promoting adherence to ART. This article described the results of a pilot RCT comparing Positive STEPS (Positive Strategies to Enhance Problem-solving Skills), an intervention comprised of daily text message reminders and video vignettes, to a standard of care condition (53). Stigma was described in the introduction as conceptually important but was not measured in the context of the intervention. The pilot study demonstrated promising results for ART adherence, with participants randomized to Positive STEPS reporting a significantly greater increase in ART than participants randomized to the standard of care (53).

\section{Discussion}

Our review of the literature identified several types of mHealth interventions that engage youth to reduce stigma as a way to improve HIV-related outcomes. These interventions included telehealth $(47,49)$, simulation video games (43), motion comics (44), smartphone appdelivered risk reduction (51), social media forums $(38,42)$, online video campaigns (50), video vignettes (53), and a computerized behavioral learning module (46). Some of these interventions were completely deliverable over the internet [e.g., SOLVE (43), PrEPTECH (49), HMP $(38,42)$, Project Moxie $(47,48)$, and MyPEEPS Mobile (51)], reflecting the ability of mHealth to overcome barriers to service utilization by reaching participants directly. Other interventions were implemented as a complement to face-to-face interventions. The video vignettes and text message reminders used in the Positive STEPS intervention to promote ART adherence, for example, were used to supplement five in-person intervention sessions with a master's level counselor (53). Similarly, the KIU intervention included the computerized behavioral learning module within a broader multi-component, in-person intervention that included biological screenings (46).

We found only two RCTs of mHealth interventions for youth in the US that aimed to reduce stigma as a way to improve HIV-related outcomes $(42,43)$. It is notable that both of these RCTs (SOLVE and HealthMpowerment) aimed to prevent primary HIV acquisition by reducing condomless anal intercourse. This suggests there is room for innovation applying mHealth tools across the HIV prevention and care continuum and still significant work to be done to move from pilot studies to effectiveness trials and wide-scale implementation and dissemination. Our findings echo calls from previous reviews for planning of future scale-up and sustainability during initial preparation and technology development of pilot studies (54).

Two of the included studies analyzing data from the RCT evaluating the HealthMpowerment intervention $(38,42)$ illustrated the opportunities mHealth interventions provide to measure and evaluate stigma and behavior change by analyzing detailed user engagement paradata, such as level of engagement in stigma-related conversations and associated health outcomes (55). As exemplified in these studies, an important additional advantage of mHealth for stigma interventions is the potential for textual analysis of user-generated content and correlation with behavior change over time (42).

Our review also documented a lack of studies addressing outcomes further along the HIV care cascade. In fact, our review only identified one study promoting adherence to ART and we did not identify any published studies using mHealth to reduce stigma as a way to improve care engagement or increase viral suppression among youth. With recent clinical trials proving the concept of undetectable $=$ untransmittable $(\mathrm{U}=\mathrm{U})(56)$, mHealth interventions have an opportunity to promote messages of $\mathrm{U}=\mathrm{U}$ to help combat stigma in interventions targeting outcomes along the care continuum. As mHealth interventions move further along this care continuum, a potential challenge will be the reliance on existing, locallyavailable health care resources for linkage to care and follow-up as these locally-available resources may not be youth-friendly, accepting, or gender-affirming (47).

The literature we reviewed ranged in the types of stigma(s) addressed in the interventions. A few interventions, like HealthMpowerment $(38,42,45)$ and Project Moxie $(47,48)$, 
focused on addressing multiple forms of stigma including sexual minority-, race/ethnicity-, and HIV-related stigma. Notably, several papers acknowledged the need to address multiple, intersecting forms of stigma in their interventions. For example, the authors of the SOLVE intervention, which successfully reduced sexual stigma among MSM enrolled in their online RCT, acknowledged financial constraints that limited their ability to develop storylines to address other intersecting stigmas that their participants may have been facing (43). Related to this point, participants in focus group discussions assessing an online video campaign designed to increase knowledge of PrEP (50), discussed the need to have materials that reflect the composition of the target audience, with Black participants noting the need to have representation of Black MSM in future programs.

Of note, some of the interventions in this review focused on addressing stigma associated with HIV testing or PrEP use without explicitly targeting additional stigmas often experienced by the populations reached. For example, the KIU intervention aimed to reduce the stigma associated with HIV testing by incorporating HIV prevention and sexual heath into a broader health promotion campaign for young Black men (46). While KIU was intended primarily for non-gay-identified men, the computerized learning module included a scene showing two men in a sexual encounter. O'Donnell et al. discussed underestimating the extent their participants would react negatively to this scene. The authors noted that the KIU intervention was likely limited as a stigma reduction intervention though it could be completed by other community-wide efforts to address stigmatizing attitudes towards MSM. Similarly, the PrEPTECH intervention sought to reduce stigma associated with PrEP use/initiation by increasing PrEP access through the telehealth intervention, though the intervention did not explicitly appear to target other forms of stigma that may have inhibited PrEP initiation among the young MSM of color in the study (49).

Our scoping review informs several recommendations for future research. First, given the role of stigma across the HIV prevention and care continuum and the negative impact on well-being among youth, we recommend concerted efforts to develop theoretically-grounded interventions that prioritize and measure changes in stigma as an important outcome. It is critical that these interventions be based on theoretical frameworks that explicitly incorporate stigma. Future interventions are encouraged to utilize frameworks that differentiate between mechanisms through which differing forms of stigma operate (57). We also echo calls for robust trials with longitudinal designs and mediation analyses to provide evidence of causal effects (10). This will allow us to better understand the mechanisms of stigma reduction in the improvement of clinical HIV outcomes among youth.

Next, we need stigma-reduction interventions that target HIV-related stigma impacting youth at the institutional, community, and structural levels of influence. Stigma operates on multiple social ecological levels and negatively impacts health outcomes among youth (58), yet stigma interventions primarily target the individual and interpersonal levels $(26,59)$. There is a notable lack of interventions addressing stigma perpetuated at community, organizational, and structural levels by health care providers, health care systems, schools, and governments (60). This gap was supported by the lack of studies identified through this review. Limitations in resources and ability to design interventions at these broader levels will continue to hamper efforts to address stigma beyond the interpersonal level, but efforts must be made to incorporate stigma at higher levels of the social ecological model into the conceptual framework and study design of future stigma interventions. More attention must also be paid to the development and evaluation of multi-level interventions that simultaneously target these intersecting and multiple level stigmas in research design and intervention development.

Finally, mHealth-delivered stigma interventions are encouraged to apply a resilience-based conceptual framework to further aid in the destigmatization of multiple minority youth at risk for HIV or poor HIV-related health outcomes. HIV risk and poor health outcomes for YHIV are most often conceptualized as individual-level behaviors and sociodemographic characteristics (5,61-63). This approach reinforces negative stereotypes about populations vulnerable to HIV $(64,65)$ and furthers stigmatization. A resilience-based framework for stigma interventions focuses instead on marginalized individuals' abilities to overcome barriers to prevention and care $(38,66)$. mHealth interventions targeting youth through social networks have an opportunity to amplify resilience processes among peer networks of youth to promote self-acceptance and provide support for youth experiencing or anticipating stigma, promoting wider resilience against adversity and stigma than interventions acting on the individual level alone (38).

This review has several limitations. Our search strategy identified studies using keywords and $\mathrm{MeSH}$ terms related to stigma, and may have missed interventions that conceptualized stigma as part of the theoretical model, but 
not as a main outcome. The authors are aware of at least three such interventions $(52,67,68)$. We also restricted our review to studies conducted in the US. While stigma is a global phenomenon, it is culturally-varied and based on different attributes in differing settings. Further studies should explore variations across cultures in the impact of mHealth stigma-reduction interventions targeting youth along the HIV continuum. Finally, our review was limited to published literature and mHealth is a rapidly evolving field with many studies underway and in development. The authors are aware of several interventions in development, such as the extension of the HealthMpowerment RCT (69), which focuses explicitly on stigma reduction using a tailored mobile app for youth with and without HIV. Further, a number of currently underway mHealth RCTs supported by the National Institutes of Health funded US. Adolescent Medicine Trials Network for HIV/AIDS Interventions (ATN) will address stigma through a variety of approaches including mail-based HIV/STI testing, remote PrEP access, remote couples-based voluntary counseling and testing, and social connectedness apps for YHIV and transgender and gender non-conforming youth at-risk for $\operatorname{HIV}(70,71)$.

In conclusion, the broad and rapidly evolving field of mHealth presents new opportunities to deliver content for stigma interventions for youth and provides space for youth to contribute to anti-stigma efforts. As a vehicle for intervention implementation and evaluation, $\mathrm{mHealth}$ offers unique advantages to address the complex intersecting stigma barriers along the HIV continuum by dynamic tailoring based on evolving individual needs. Effectively addressing stigma as a barrier to improved HIV-related outcomes is at the core of the Department of Health and Human Services' Ending the HIV Epidemic: A Plan for America initiative $(72,73)$. mHealth is uniquely poised to address stigma and its role in perpetuating disparities in the HIV epidemic among US youth.

\section{Acknowledgments}

Funding: Research reported in this publication was supported by the National Institute of Minority Health and Health Disparities under Award Number R01MD013623 and the Eunice Kennedy Shriver National Institute of Child Health and Human Development (NICHD) under Award Number U19HD089881. MIM was also supported by the National Institute of Mental Health of the National Institutes of Health under Award Number K01MH118072.

\section{Footnote}

Provenance and Peer Review: This article was commissioned by the Guest Editor (Lisa Hightow-Weidman) for the series "Technology-based Interventions in HIV Prevention and Care Continuum among American Youth" published in $m$ Health. The article has undergone external peer review.

Conflicts of Interest: All authors have completed the ICMJE uniform disclosure form. (available at http://dx.doi. org/10.21037/mhealth-20-68). MIM reports grants from the Duke University School of Nursing during the conduct of the study. LBHW reports grants from University of North Carolina at Chapel Hill during the conduct of the study. KEM reports grants from University of North Carolina at Chapel Hill during the conduct of the study. The authors have no conflicts of interest to declare.

Ethical Statement: The authors are accountable for all aspects of the work in ensuring that questions related to the accuracy or integrity of any part of the work are appropriately investigated and resolved.

Disclosure: The content is solely the responsibility of the authors and does not necessarily represent the official views of the National Institutes of Health.

Open Access Statement: This is an Open Access article distributed in accordance with the Creative Commons Attribution-NonCommercial-NoDerivs 4.0 International License (CC BY-NC-ND 4.0), which permits the noncommercial replication and distribution of the article with the strict proviso that no changes or edits are made and the original work is properly cited (including links to both the formal publication through the relevant DOI and the license). See: https://creativecommons.org/licenses/by-nc-nd/4.0/.

\section{References}

1. Centers for Disease Control and Prevention. HIV Surveillance Report, 2018 (Preliminary) Atlanta, GA: Centers for Disease Control and Prevention. Available online: http://www.cdc.gov/hiv/library/reports/hivsurveillance.html

2. Centers for Disease Control and Prevention. HIV Among Youth Atlanta, GA: Centers for Disease Control and Prevention. Available online: https://www.cdc.gov/hiv/ group/age/youth/index.html 
3. Centers for Disease Control and Prevention. Selected National HIV Prevention and Care Outcomes Atlanta, GA: Centers for Disease Control and Prevention. Available online: https://www.cdc.gov/hiv/pdf/library/slidesets/cdchiv-prevention-and-care-outcomes.pdf

4. Gamarel KE, Nelson KM, Stephenson R, et al. Anticipated HIV Stigma and Delays in Regular HIV Testing Behaviors Among Sexually-Active Young Gay, Bisexual, and Other Men Who Have Sex with Men and Transgender Women. AIDS Behav 2018;22:522-30.

5. Mustanski B, Morgan E, D'Aquila R, et al. Individual and Network Factors Associated With Racial Disparities in HIV Among Young Men Who Have Sex With Men: Results From the RADAR Cohort Study. J Acquir Immune Defic Syndr 2019;80:24-30.

6. Rosengren AL, Menza TW, LeGrand S, et al. Stigma and Mobile App Use Among Young Black Men Who Have Sex With Men. AIDS Educ Prev 2019;31:523-37.

7. Jaiswal J, Griffin M, Singer SN, et al. Structural Barriers to Pre-exposure Prophylaxis Use Among Young Sexual Minority Men: The P18 Cohort Study. Curr HIV Res 2018;16:237-49.

8. Peng P, Su S, Fairley CK, et al. A Global Estimate of the Acceptability of Pre-exposure Prophylaxis for HIV Among Men Who have Sex with Men: A Systematic Review and Meta-analysis. AIDS Behav 2018;22:1063-74.

9. Valverde E, Rodriguez A, White B, et al. Understanding the Association of Internalized HIV Stigma with Retention in HIV Care. J HIV AIDS 2018. doi: 10.16966/23805536.159 .

10. Turan B, Hatcher AM, Weiser SD, et al. Framing Mechanisms Linking HIV-Related Stigma, Adherence to Treatment, and Health Outcomes. Am J Public Health 2017;107:863-9.

11. Rueda S, Mitra S, Chen S, et al. Examining the associations between HIV-related stigma and health outcomes in people living with HIV/AIDS: a series of meta-analyses. BMJ Open 2016;6:e011453.

12. Katz IT, Ryu AE, Onuegbu AG, et al. Impact of HIVrelated stigma on treatment adherence: systematic review and meta-synthesis. J Int AIDS Soc 2013;16:18640.

13. Arnold EA, Rebchook GM, Kegeles SM. 'Triply cursed': racism, homophobia and HIV-related stigma are barriers to regular HIV testing, treatment adherence and disclosure among young Black gay men. Cult Health Sex 2014;16:710-22

14. Miller RL, Lee KS, Chiaramonte D, et al. Youth health outcomes from the Connect-to-Protect Coalitions to prevent adolescent HIV infections. Vulnerable Child Youth Stud 2018;13:142-57.

15. Miller RL, Strzyzykowski T, Lee KS, et al. Structural Effects on HIV Risk Among Youth: A Multi-level Analysis. AIDS Behav 2018;22:3451-67.

16. Marti-Pastor M, Ferrer M, Alonso J, et al. Association of Enacted Stigma with Depressive Symptoms Among Gay and Bisexual Men Who Have Sex with Men: Baltimore, 2011 and 2014. LGBT Health 2020;7:47-59.

17. Turan B, Budhwani H, Fazeli PL, et al. How Does Stigma Affect People Living with HIV? The Mediating Roles of Internalized and Anticipated HIV Stigma in the Effects of Perceived Community Stigma on Health and Psychosocial Outcomes. AIDS Behav 2017;21:283-91.

18. Casale M, Boyes M, Pantelic M, et al. Suicidal thoughts and behaviour among South African adolescents living with HIV: Can social support buffer the impact of stigma? J Affect Disord 2019;245:82-90.

19. Centers for Disease Control and Prevention. HIV and African American Gay and Bisexual Men: Atlanta, GA. Available online: https://www.cdc.gov/hiv/group/msm/ bmsm.html

20. Centers for Disease Control and Prevention. Diagnoses of HIV Infection in the United States and Dependent Areas, 2017 Atlanta, GA: Centers for Disease Control and Prevention. Available online: https://www.cdc.gov/hiv/pdf/ library/reports/surveillance/cdc-hiv-surveillance-report2017-vol-29.pdf

21. Earnshaw VA, Bogart LM, Dovidio JF, et al. Stigma and racial/ethnic HIV disparities: moving toward resilience. Am Psychol 2013;68:225-36.

22. Arscott J, Humphreys J, Merwin E, et al. "That Guy is Gay and Black. That's a Red Flag." How HIV Stigma and Racism Affect Perception of Risk Among Young Black Men Who Have Sex with Men. AIDS Behav 2020;24:173-84.

23. Lichtenstein B, Kay ES, Klinger I, et al. Ricky and Lucy: gender stereotyping among young Black men who have sex with men in the US Deep South and the implications for HIV risk in a severely affected population. Cult Health Sex 2018;20:351-65.

24. Becasen JS, Denard CL, Mullins MM, et al. Estimating the Prevalence of HIV and Sexual Behaviors Among the US Transgender Population: A Systematic Review and MetaAnalysis, 2006-2017. Am J Public Health 2019;109:e1-e8.

25. Magno L, Silva L, Veras MA, et al. Stigma and discrimination related to gender identity and vulnerability to HIV/AIDS among transgender women: a systematic 
review. Cad Saude Publica 2019;35:e00112718.

26. Rao D, Elshafei A, Nguyen M, et al. A systematic review of multi-level stigma interventions: state of the science and future directions. BMC Med 2019;17:41.

27. Rao D, Frey S, Ramaiya M. eHealth for Stigma Reduction Efforts Designed to Improve Engagement in Care for People Living with HIV. Curr HIV/AIDS Rep 2018;15:397-402.

28. Andersson GZ, Reinius M, Eriksson LE, et al. Stigma reduction interventions in people living with HIV to improve health-related quality of life. Lancet HIV 2020;7:e129-40.

29. Hartog K, Hubbard CD, Krouwer AF, et al. Stigma reduction interventions for children and adolescents in low- and middle-income countries: Systematic review of intervention strategies. Soc Sci Med 2020;246:112749.

30. McAteer CI, Truong NT, Aluoch J, et al. A systematic review of measures of HIV/AIDS stigma in paediatric $\mathrm{HIV}$-infected and HIV-affected populations. J Int AIDS Soc 2016;19:21204.

31. Grossman CI, Stangl AL. Editorial: Global action to reduce HIV stigma and discrimination. J Int AIDS Soc 2013;16:18881.

32. Amico KR. Evidence for Technology Interventions to Promote ART Adherence in Adult Populations: a Review of the Literature 2012-2015. Curr HIV/AIDS Rep 2015;12:441-50.

33. Burk J, Park M, Saewyc EM. A Media-Based School Intervention to Reduce Sexual Orientation Prejudice and Its Relationship to Discrimination, Bullying, and the Mental Health of Lesbian, Gay, and Bisexual Adolescents in Western Canada: A Population-Based Evaluation. Int J Environ Res Public Health 2018;15:2447.

34. Holloway IW, Rice E, Gibbs J, et al. Acceptability of smartphone application-based HIV prevention among young men who have sex with men. AIDS Behav 2014;18:285-96.

35. Holloway IW, Winder TJ, Lea CH III, et al. Technology Use and Preferences for Mobile Phone-Based HIV Prevention and Treatment Among Black Young Men Who Have Sex With Men: Exploratory Research. JMIR Mhealth Uhealth 2017;5:e46.

36. Bauermeister JA, Pingel ES, Jadwin-Cakmak L, et al. Acceptability and preliminary efficacy of a tailored online HIV/STI testing intervention for young men who have sex with men: the Get Connected! program. AIDS Behav 2015;19:1860-74.

37. Muessig KE, LeGrand S, Horvath KJ, et al. Recent mobile health interventions to support medication adherence among HIV-positive MSM. Curr Opin HIV AIDS 2017;12:432-41.

38. Barry MC, Threats M, Blackburn NA, et al. "Stay strong! keep ya head up! move on! it gets better!!!!": resilience processes in the healthMpowerment online intervention of young black gay, bisexual and other men who have sex with men. AIDS Care 2018;30:S27-S38.

39. Pew Research Center. Teens, Social media, and technology 2018. Available online: https://www.pewresearch.org/ internet/2018/05/31/teens-social-media-technology-2018/

40. Thapa S, Hannes K, Cargo M, et al. Stigma reduction in relation to HIV test uptake in low- and middleincome countries: a realist review. BMC Public Health 2018;18:1277.

41. Hatzenbuehler ML, Phelan JC, Link BG. Stigma as a fundamental cause of population health inequalities. Am J Public Health 2013;103:813-21.

42. Bauermeister JA, Muessig KE, LeGrand S, et al. HIV and Sexuality Stigma Reduction Through Engagement in Online Forums: Results from the HealthMPowerment Intervention. AIDS Behav 2019;23:742-52.

43. Christensen JL, Miller LC, Appleby PR, et al. Reducing shame in a game that predicts HIV risk reduction for young adult MSM: a randomized trial delivered nationally over the Web. J Int AIDS Soc 2013;16:18716.

44. Willis LA, Kachur R, Castellanos TJ, et al. Developing a Motion Comic for HIV/STD Prevention for Young People Ages 15-24, Part 2: Evaluation of a Pilot Intervention. Health Commun 2018;33:229-37.

45. Hightow-Weidman LB, LeGrand S, Muessig KE, et al. A Randomized Trial of an Online Risk Reduction Intervention for Young Black MSM. AIDS Behav 2019;23:1166-77.

46. O'Donnell L, Bonaparte B, Joseph H, et al. Keep It Up: development of a community-based health screening and HIV prevention strategy for reaching young African American men. AIDS Educ Prev 2009;21:299-313.

47. Stephenson R, Metheny N, Sharma A, et al. Providing Home-Based HIV Testing and Counseling for Transgender Youth (Project Moxie): Protocol for a Pilot Randomized Controlled Trial. JMIR Res Protoc 2017;6:e237.

48. Stephenson R, Todd K, Kahle E, et al. Project Moxie: Results of a Feasibility Study of a Telehealth Intervention to Increase HIV Testing Among Binary and Nonbinary Transgender Youth. AIDS Behav 2020;24:1517-30.

49. Refugio ON, Kimble MM, Silva CL, et al. Brief Report: PrEPTECH: A Telehealth-Based Initiation Program for 
HIV Pre-exposure Prophylaxis in Young Men of Color Who Have Sex With Men. A Pilot Study of Feasibility. J Acquir Immune Defic Syndr 2019;80:40-5.

50. Thomann M, Grosso A, Zapata R, et al. 'WTF is PrEP?': attitudes towards pre-exposure prophylaxis among men who have sex with men and transgender women in New York City. Cult Health Sex 2018;20:772-86.

51. Schnall R, Kuhns LM, Hidalgo MA, et al. Adaptation of a Group-Based HIV RISK Reduction Intervention to a Mobile App for Young Sexual Minority Men. AIDS Educ Prev 2018;30:449-62.

52. Amico KR, Dunlap A, Dallas R, et al. Triggered Escalating Real-Time Adherence Intervention to Promote Rapid HIV Viral Suppression Among Youth Living With HIV Failing Antiretroviral Therapy: Protocol for a Triggered Escalating Real-Time Adherence Intervention. JMIR Res Protoc 2019;8:e11416.

53. Mimiaga MJ, Bogart LM, Thurston IB, et al. Positive Strategies to Enhance Problem-Solving Skills (STEPS): A Pilot Randomized, Controlled Trial of a Multicomponent, Technology-Enhanced, Customizable Antiretroviral Adherence Intervention for HIV-Infected Adolescents and Young Adults. AIDS Patient Care STDS 2019;33:21-4.

54. Mulawa MI, LeGrand S, Hightow-Weidman LB. eHealth to Enhance Treatment Adherence Among Youth Living with HIV. Curr HIV/AIDS Rep 2018;15:336-49.

55. Bauermeister JA, Golinkoff JM, Muessig KE, et al. Addressing engagement in technology-based behavioural HIV interventions through paradata metrics. Curr Opin HIV AIDS 2017;12:442-6.

56. Eisinger RW, Dieffenbach CW, Fauci AS. HIV Viral Load and Transmissibility of HIV Infection: Undetectable Equals Untransmittable. JAMA 2019;321:451-2.

57. Earnshaw VA, Smith LR, Chaudoir SR, et al. HIV stigma mechanisms and well-being among PLWH: a test of the HIV stigma framework. AIDS Behav 2013;17:1785-95.

58. Oldenburg CE, Perez-Brumer AG, Hatzenbuehler ML, et al. State-level structural sexual stigma and HIV prevention in a national online sample of HIV-uninfected MSM in the United States. AIDS 2015;29:837-45.

59. Philbin MM, Parker CM, Parker RG, et al. The Promise of Pre-Exposure Prophylaxis for Black Men Who Have Sex with Men: An Ecological Approach to Attitudes, Beliefs, and Barriers. AIDS Patient Care STDS 2016;30:282-90.

60. Sekoni AO, Gale NK, Manga-Atangana B, et al. The effects of educational curricula and training on LGBTspecific health issues for healthcare students and professionals: a mixed-method systematic review. J Int
AIDS Soc 2017;20:21624.

61. Patel P, Borkowf CB, Brooks JT, et al. Estimating peract HIV transmission risk: a systematic review. Aids 2014;28:1509-19.

62. Baggaley RF, White RG, Boily MC. HIV transmission risk through anal intercourse: systematic review, meta-analysis and implications for HIV prevention. Int J Epidemiol 2010;39:1048-63.

63. Anderson PL, Glidden DV, Liu A, et al. Emtricitabinetenofovir concentrations and pre-exposure prophylaxis efficacy in men who have sex with men. Sci Transl Med 2012;4:151ra125.

64. Rowan D, DeSousa M, Randall EM, et al. "We're just targeted as the flock that has HIV": health care experiences of members of the house/ball culture. Soc Work Health Care 2014;53:460-77.

65. Herrick AL, Stall R, Chmiel JS, et al. It gets better: resolution of internalized homophobia over time and associations with positive health outcomes among MSM. AIDS Behav 2013;17:1423-30.

66. DiFulvio GT. Sexual minority youth, social connection and resilience: from personal struggle to collective identity. Soc Sci Med 2011;72:1611-7.

67. Muessig KE, Knudtson KA, Soni K, et al. 'I Didn't Tell You Sooner Because I Didn't Know How to Handle it Myself'. Developing a Virtual Reality Program to Support HIV-Status Disclosure Decisions. Digit Cult Educ 2018;10:22-48.

68. LeGrand S, Knudtson K, Benkeser D, et al. Testing the Efficacy of a Social Networking Gamification App to Improve Pre-Exposure Prophylaxis Adherence (P3: Prepared, Protected, emPowered): Protocol for a Randomized Controlled Trial. JMIR Res Protoc 2018;7:e10448.

69. ClinicalTrials.gov Identifier: NCT03678181. Increasing Engagement and Improving HIV Outcomes Via HealthMPowerment. Available online: https:// clinicaltrials.gov/ct2/show/NCT03678181

70. University of North Carolina/Emory Center for Innovative Technology (iTech) Protocols. Available online: https://itechnetwork.org/protocols/

71. Hightow-Weidman LB, Muessig K, Rosenberg E, et al. University of North Carolina/Emory Center for Innovative Technology (iTech) for Addressing the HIV Epidemic Among Adolescents and Young Adults in the United States: Protocol and Rationale for Center Development. JMIR Res Protoc 2018;7:e10365.

72. Fauci AS, Redfield RR, Sigounas G, et al. Ending the 
HIV Epidemic: A Plan for the United States. JAMA 2019;321:844-5.

73. Birx DL, Giroir, B.P. World AIDS Day 2019 Ending the

doi: $10.21037 /$ mhealth-20-68

Cite this article as: Mulawa MI, Rosengren AL, Amico KR, Hightow-Weidman LB, Muessig KE. mHealth to reduce HIVrelated stigma among youth in the United States: a scoping review. mHealth 2021;7:35.
HIV/AIDS Epidemic: Community by Community 2019. Available online: https://www.hhs.gov/blog/2019/11/25/ world-aids-day-2019-ending-the-hiv-aids-epidemic.html 
Supplementary

Search Terms: mHealth for HIV Stigma Reduction Among Youth

Database: PubMed

Data search completed: 2/18/2020

\begin{tabular}{|c|c|c|}
\hline Set & Search & Results \\
\hline$\# 2$ & $\begin{array}{l}\text { HIV Infections [MeSH] or HIV [MeSH] OR hiv1 [TIAB] OR hiv2 [TIAB] OR "human immunodeficiency } \\
\text { virus" [TIAB] OR "human immune deficiency virus" [TIAB] }\end{array}$ & 333,253 \\
\hline \multicolumn{3}{|l|}{ HIV } \\
\hline \#3 Stigma & $\begin{array}{l}\text { "Social Stigma" [MeSH] OR Shame [MeSH] OR "Social Discrimination" [MeSH] OR Prejudice [MeSH] } \\
\text { OR Homophobia [MeSH] OR Racism [MeSH] OR Stigma* [TIAB] OR Shame [TIAB] OR Discriminat* } \\
\text { [TIAB] OR Prejudice* [TIAB] OR Distrust [TIAB] OR Homophobia [TIAB] OR Homonegativity [TIAB] OR } \\
\text { Transphobia [TIAB] OR Racism [TIAB] OR "Racial discrimination" [TIAB] }\end{array}$ & 292,484 \\
\hline \#4 Youth & \#1 AND \#2 AND \#3 AND \#4 & 310 \\
\hline
\end{tabular}

